\title{
Effects of gestational subclinical hypothyroidism and TPO-Ab on pregnancy outcomes
}

\author{
Wen Wei ${ }^{1}$, Ping Liu*2, Hairong Guo ${ }^{2}$ \\ ${ }^{1}$ Inner Mongolia Medical University, Hohehot, Inner Mongolia, China \\ ${ }^{2}$ Department of Obstetrics and Gynecology, the Third Affiliated Hospital of Inner Mongolia Medical University, Baotou, Inner \\ Mongolia, China
}

Received: March 27, 2018

DOI: $10.14725 /$ dcc.v5n2p20
Accepted: May 5, 2018

URL: http://dx.doi.org/10.14725/dcc.v5n2p20

\begin{abstract}
The prevalence of gestational subclinical hypothyroidism has been increasing with years, and it has become one of the common diseases happened to women during pregnancy in China. Gestational subclinical hypothyroidism can not only increase the incidence of adverse pregnancy outcomes, but also have a negative impact on the development of the offspring. Therefore, it is necessary to make an early detection, diagnosis and treatment.
\end{abstract}

Key Words: Subclinical hypothyroidism, Thyroid peroxidase antibody, Pregnancy outcomes

Subclinical hypothyroidism refers to a disease that patients have a higher level of thyroid stimulating hormone (TSH) than the normal range, with a normal serum free thyroxine $\left(\mathrm{FT}_{4}\right)$ level. ${ }^{[1]}$ Gestation period is the most special one in a woman's life. During pregnancy, thyroid hormones are very important to a pregnant woman and her fetus, and the pregnant satisfy a drive for thyroid hormones by regulating the axis of hypothalamus-hypophysis-thyroid. Changes in maternal thyroid hormone level during the gestation period may be related to the elevated maternal human chorionic gonadotropin (hCG) and thyroxine-binding globulin (TBG) levels. ${ }^{[2]}$ If the pregnant cannot adapt to this type of changes, thyroid dysfunction will probably occur. In that case, subclinical hypothyroidism will be transformed into clinical hypothyroidism, especially in patients with positive thyroid peroxidase antibodies (TPO-Ab). In a foreign epidemiological investigation, it was found that ${ }^{[3]}$ the incidence of gestational subclinical hypothyroidism was $2 \%$ to $3 \%$; and domestic studies showed that in the first trimester of pregnancy, the incidence of subclinical hypothyroidism was $5.27 \% .{ }^{[4]}$ In recent years, the prevalence of gestational subclinical hypothyroidism has been increasing with years, and it has become one of the common diseases happened to women during pregnancy in China. Gestational subclinical hypothyroidism can not only increase the incidence of adverse pregnancy outcomes, but also have a negative impact on the development of the offspring. Therefore, it is necessary to make an early detection, diagnosis and treatment. ${ }^{[5]}$

\section{Diagnostic criteria of gestational sub- clinical hypothyroidism and TPO-Ab positivity}

According to "Chinese Guidelines for the Diagnosis and Management of Thyroid Diseases During Pregnancy and Postpartum" established by Chinese Medical Association in

\footnotetext{
*Correspondence: Ping Liu; E-mail: liupingbg@126.com; Address: Department of Obstetrics and Gynecology, the Third Affiliated Hospital of Inner Mongolia Medical University, Baotou, Inner Mongolia, China.
} 
2012, the diagnostic criteria of gestational subclinical hypothyroidism are as follows: The level of TSH is above the upper limit of pregnancy-specific reference range $\left(97.5^{\text {th }}\right.$ percentile), while the level of $\mathrm{FT}_{4}$ still falls into the reference range $\left(2.5^{t h}-97.5^{t h}\right.$ percentile). For example, if the level of serum TSH is more than $10 \mathrm{mIU} / \mathrm{L}$, it will be diagnosed as clinical hypothyroidism no matter the level of $\mathrm{FT}_{4}$ is decreased or not.

The diagnostic criteria of TPO-Ab positivity are as follows: ${ }^{[6]}$ The TPO-Ab level is higher than the upper limit of the reference range provided by the kit; simple TPO-Ab positivity refers to the decrease of $\mathrm{FT}_{4}$ level with no increase of TSH level.

\section{Effect of gestational subclinical hy- pothyroidism on pregnancy outcomes}

\subsection{Gestational diabetes mellitus (GDM)}

GDM refers to a condition in which a woman with normal glucose metabolism or abnormal glucose tolerance develops impending or diagnosed diabetes during pregnancy; ${ }^{[7]}$ the incidence is $18.7 \% .^{[8]}$ Thyroid hormone is a type of important hormone against the action of insulin. It can not only accelerate the decomposition of insulin, but also change the sensitivity of the body to insulin. Normally, insulin in the human body is in a state of dynamic equilibrium with hormones that are against insulin. Therefore, blood glucose is maintained in a normal range. With the increase in the pregnant month, the demand from the pregnant for thyroid hormone is also gradually increased. In order to adapt to this type of change, thyroid gland regulates the level of thyroid hormone in the pregnant through TBG, hCG and fetal thyroid gland. During pregnancy, insulin resistance exists in the pregnant, and this physiological state maintains glucose metabolism in the body by a way that islet $\beta$ cells secrete insulin. ${ }^{[9,10]}$ If the balance of glucose metabolism fails to be maintained, it will be developed into GDM. After excluding susceptibility factors such as genetics and age, if the level of TSH is increased, the risk for the development of GDM is also increased. [11] Moreover, high blood glucose and osmotic diuresis are main causes of polyhydramnios in the pregnant.

\subsection{Hypertensive disorder complicating pregnancy (HDCP)}

The pathogenesis of HDCP remains unclear up to now, and it is thought to be related to the damage of vascular endothelial cells, excessive activation of inflammatory immunity, insufficient remodeling of uterine spiral arterioles, insulin resistance and genetic factors. Thyroid hormone has an obviously significant effect on the cardiovascular sys- tem. The cardiac output is increased in patients with hyperthyroidism, with peripheral vascular resistance decreased. This pattern is the opposite of the one in patients with hypothyroidism. Therefore, the cardiac index is decreased in pregnant women with hypothyroidism, with cardiac output decreased, peripheral vascular resistance increased and blood pressure elevated. Although gestational subclinical hypothyroidism only increases TSH level, it still increases the risk of cardiovascular diseases. ${ }^{[12]}$ The higher TSH level is, the higher systolic blood pressure and diastolic blood pressure are. It is indicated that TSH level is positively correlated to blood pressure. ${ }^{[13]}$ Subclinical hypothyroidism is involved in the formation and development of atherosclerosis by aggravating vascular endothelial cell dysfunction, and atherosclerosis can result in luminal stenosis or even occlusion of spiral arterioles, leading to a lower placental blood flow and causing a series of HDCP symptoms.

\subsection{Preterm birth, miscarriage and newborn mental development}

An abnormal level of thyroid hormones can lead to abnormal excitability in nervous system, cardiovascular system, muscle etc. It can also give rise to the increase in body oxygen consumption and the release of hormones such as norepinephrine and angiotensins, resulting in microvasospasm and stronger uterine contraction. ${ }^{[14]}$ Studies ${ }^{[15]}$ have found that the elevated level of TSH in pregnant women would increase the incidence of miscarriage, preterm birth and fetal distress. Therefore, clinicians should improve the correct understanding and treatment of gestational subclinical hypothyroidism. In the first trimester of pregnancy, the fetus cannot synthesize thyroid hormone by itself. Nevertheless, the first trimester of pregnancy is the most critical period of fetal brain development. If thyroid hormone is lacking, it will seriously affect the development of fetal nerve cells. The lack of maternal thyroid hormone can cause fetal brain dysplasia and affect newborn babies' intelligence. Pop $^{[16]}$ reported that, gestational subclinical hypothyroidism affects fetal brain development, resulting in lower intelligence grade in pregnant women's offspring.

\section{Effects of TPO-Ab during gestation pe- riod on pregnancy}

TPO-Ab is an important immune indicator and closely related to gestational subclinical hypothyroidism. Most patients with gestational subclinical hypothyroidism showed positive TPO-Ab, however, there are also some patients only showing positive TPO-Ab with normal thyroid function (confirmed by tests). If TPO-Ab appears to be positive in pregnant women, it will affect fetal growth and development through the placenta. ${ }^{[17]}$ Studies $^{[18]}$ found that 
either subclinical hypothyroidism or subclinical hypothyroidism combined with TPO-Ab positivity would result in gestational complications, and make the risk of HDCP, placental abruption, preterm birth, miscarriages increased by 2 to 3 times. Some studies also ${ }^{[19]}$ have shown that TPO-Ab positivity is closely associated with recurrent spontaneous abortion. In the research conducted by Gleicher ${ }^{[20]}$ it is showed that thyroid autoantibodies have some influences on the occurrence of eclampsia and preeclampsia. The probability of adverse pregnancy outcomes is significantly higher in pregnant women with positive TPO-Ab. After thyroid hormone replacement therapy is given, pregnancy outcomes can be significantly improved in pregnant women with gestational subclinical hypothyroidism combined with TPO-Ab positivity. Therefore, hormone replacement therapy acts as a very important role. Karakosta et al. ${ }^{[21]}$ considered that the probability of glycometabolism abnormality in pregnant

\section{References}

[1] $\mathrm{Xu} \mathrm{CH}$. Correlation analysis of gestational hypothyroidism and pregnancy outcomes. Maternal and Child Health Care of China. 2016; 31(7): 1376-1377.

[2] Qin F. Influence of thyroid function during pregnancy on pregnancy outcome. Chinese and Foreign Medical Research. 2016; 14(14): 107-109.

[3] Abalovich M, Amino N, Barbour LA, et al. Management of thyroid dysfunction during pregnancy and postpartum: an Endocrine Society Clinical Practice Guideline. J Clin Endocrinol Metab. 2007; 92(8 Suppl): s1-s47. PMid: 17948378. https://doi.org/10.1210/ jc. 2007-0141

[4] Fan XW. Changes in thyroid function during pregnancy and its influence on the fetus. Journal of International Obstetrics and Gynecolog. 2015; 42(3): 330-334.

[5] Zhu YF, Shi MH, Lv MD, et al. Relationship between subclinical thyroid dysfunction and pregnancy outcome. Zhejiang Practical Medicine. 2015; 20(5): 323-324, 331.

[6] Stagnaro-Green A, Abalovich M, Alexander E, et al. Guidelines of the American Thyroid Association for the diagnosis and management of thyroid disease during pregnancy and postpartum. Thyroid. 2011; 21(10): 1081-1125. PMid: 21787128. https://doi.org/ $10.1089 /$ thy .2011 .0087

[7] American Diabetes Association. Standards of medical care in diabetes-2011. Diabetes Care. 2011; 34(Suppl 1): S11-S61. PMid: 21193625. https://doi.org/10.2337/dc11-S011

[8] Gudović A, Spremović-Radjenović S, Lazović G, et al. Maternal autoimmune thyroid disease and pregnancy complication. Vojnosanitetski Pregled. 2010; 67(8): 617-21. PMid: 20845662. https: //doi.org/10.2298/VSP1008617G

[9] Dong Y, Wang XM. Influence of hypothyroidism on gestational diabetes mellitus. Chinese Journal of Woman and Child Health Research. 2016; 27(7): 834-837.

[10] Li WL, Wang YX. Clinical significance of serum lipids measurement in patients with gestational diabetes mellitus at late phase. China Tropical Medicine. 2016; 16(1): 80-83.

[11] Tudela CM, Casey BM, McIntire DD, et al. Relationship of subclinical thyroid disease to the incidence of gestational diabetes. Ob- women with elevated TSH level and TPO-Ab positivity was obviously improved, and was probably associated with autoimmune function. Among pregnant women with subclinical hypothyroidism and TPO-Ab negativity, the incidence of adverse pregnancy outcomes was significantly lower in the treated than the untreated.

In summary, clinicians should give thyroid function tests to pregnant women during early prenatal examination, so as to find problems in time and give an early systemic thyroid hormone replacement therapy. Reasonable treatment and regular detection of thyroid function can significantly reduce the incidence of adverse pregnancy outcomes.

\section{Conflicts of Interest Disclosure}

The authors have no conflicts of interest related to this article.

stet Gynecol. 2012; 119(5): 983-988. PMid: 22525909. https: //doi.org/10.1097/AOG.0b013e318250aeeb

[12] Harvey CB, Williams GR. Mechanism of thyroid hormone action. Thyroid. 2002; 12(6): 441-446. PMid: 12165104. https://doi. org/10.1089/105072502760143791

[13] Wilson KL, Casey BM, McIntire DD, et al. Subclinical thyroid disease and the incidence of hypertension in pregnancy. Obstet Gynecol. 2012; 119 (2 Pt 1): 315-320. PMid: 22270283. https: //doi.org/10.1097/AOG.0b013e318240de6a

[14] Sun YY, Zhao W. Indication and significance of the screening for thyroid function. Guide of China Medicine. 2016; 14(8): 86-87.

[15] Jiang F, Gao JS, Ma LK, et al. Pregnancy outcomes in patients with gestational hypothyroidism after specified hormone replacement therapy. Journal of Reproductive Medicine. 2013; 22(11): 836840.

[16] Pop VJ, Brouwers EP, Vader HL, et al. Maternal hypothyroxinaemia during early pregnancy and subsequent child development: a 3 year follow-up study. Clin Endocrinol (Oxf). 2003; 59(3): 282-288. https://doi.org/10.1046/j.1365-2265.2003.01822.x

[17] Yu W, Shi F. Influence on pregnancy outcome of 41 pregnant women with subclinical hypothyroidism after medical treatment. Modern Medicine Journal of China. 2015; 17(6): 24-26.

[18] Zhao PX. Gestational thyroid dysfunction and adverse pregnancy outcomes. Journal of Hubei University of Science: Medical Science. 2015; 29(1): 82-85.

[19] Pratt DE, Kaberlein G, Dudkiewicz A, et al. The association of antithyroid antibodies in euthyroid nonpregnant women with recurrent first trimester abortions in the next pregnancy. Fertil Steril. 1993; 60(6): 1001-1005. https://doi.org/10.1016/ S0015-0282 (16) 56400-0

[20] Gleicher N. Maternal autoimmunity and adverse pregnancy outcomes. J Autoimmun. 2014; 50(1): 83-86. PMid: 24461538. https://doi.org/10.1016/j.jaut.2013.12.009

[21] Karakosta P, Alegakis D, Georgiou V, et al. Thyroid dysfunction and autoantibodies in early pregnancy are associated with increased risk of gestational diabetes and adverse birth outcomes. J Clin Endocrinol Metab. 2012; 97(12): 4464-4472. PMid: 23015651. https://doi.org/10.1210/jc.2012-2540 